\title{
Gaps between Knowledge and Malaria Treatment Practices after Intensive Anti-Malaria Campaigns in Western Kenya: 2004-2016
}

\author{
Guofa Zhou, ${ }^{1}$ Elizabeth Hemming-Schroeder, ${ }^{1}$ Maxwell Gesuge, ${ }^{2}$ Yaw A. Afrane, ${ }^{3}$ Ming-Chieh Lee, ${ }^{1}$ Harrysone E. Atieli, ${ }^{4}$ \\ Andrew K. Githeko, ${ }^{2}$ and Guiyun Yan ${ }^{1 *}$
}

${ }^{1}$ Program in Public Health, University of California, Irvine, California; ${ }^{2}$ Centre for Global Health Research, Kenya Medical Research Institute, Kisumu, Kenya; ${ }^{3}$ College of Health Sciences, University of Ghana, Accra, Ghana; ${ }^{4}$ Department of Public Health, Maseno University, Maseno, Kenya

\begin{abstract}
Effective case management is central for malaria control, but not all of those affected by malaria have access to prompt, effective treatment. In Kenya, free malaria treatment has been implemented since 2006 . However, questions remain regarding effective treatment. We conducted cross-sectional epidemiological and questionnaire surveys in four counties in western Kenya in 2004, 2010, and 2016, and antimalarial availability surveys in 2016 . We found a significant decline in self-reported malaria cases and an improvement in knowledge of malaria prevention and treatment since 2004. Parasite prevalence declined significantly from 2004 to 2010; however, it has remained unchanged since then. Artemisinin-based combination therapies (ACTs) and sulfadoxine-pyrimethamine (SP) drugs were widely available everywhere. The proportion of ACT usage increased from none in 2004 to $48 \%$ and $69 \%$, respectively, in 2010 and 2016 , whereas SP drug usage declined from $88 \%$ in 2004 to $39 \%$ in 2010 and $27 \%$ in 2016 . During the 2016 survey, nonintermittent preventive treatment in pregnancy use of SP was common ( $20.9 \%$ of all surveyed individual treatments). In $2004,27.2 \%$ (168/617) of households sought hospital treatment alone, and this number increased to $50.6 \%$ in 2016 . The key factors affecting treatment-seeking behavior were education level, wealth index, household size, and distance to hospitals. Our results indicated that gaps in malaria case management remain and out-of-policy treatment is still a concern.
\end{abstract}

\section{INTRODUCTION}

About half of the world population is at risk of acquiring malaria, but most malarial deaths are among children younger than five years in sub-Saharan Africa. ${ }^{1}$ Prompt, effective treatment is the cornerstone of current malaria case management policy worldwide, in addition to vector control and other preventive measures. $^{2,3}$ In Kenya, as parasites have developed resistance to different drugs over time, malaria treatment policy has experienced major changes in the last two decades. Chloroquine (CQ) was effective against Plasmodium falciparum in Kenya until parasite resistance was detected in $1978 .{ }^{4}$ Because of widespread resistance, in 1998, CQ was replaced by sulfadoxinepyrimethamine (SP) as the first-line treatment for uncomplicated $P$. falciparum malaria in Kenya. ${ }^{5,6}$ For the same reason, SP was replaced in 2004 by the current recommended first-line treatment, artemisinin-based combination therapies (ACTs) of artemether-lumefantrine (AL), which were made widely available free of charge since 2006..$^{3,7-10}$ By policy, SP drugs are only allowed for intermittent preventive treatment in pregnancy (IPTp-SP) since 2006. ${ }^{3}$ However, SP drugs are widely available in private shops. ${ }^{11}$ The major changes in malaria treatment policy since 2010 were the shifts of second-line treatment of uncomplicated falciparum malaria. Specifically, for treatment failure of $A L$, the treatment changed from oral quinine (QN) to dihydroartemisinin-piperaquine (DHA-PPQ), and treatment of severe malaria changed from parenteral $\mathrm{QN}$ to parenteral artesunate.

Monitoring the availability and usage of antimalarials is essential for improving prompt access to effective malaria treatment and delaying the development of parasite resistance to antimalarial drugs. ${ }^{12}$ Artemisinin-based combination therapies

* Address correspondence to Guiyun Yan or Guofa Zhou, Program in Public Health, University of California at Irvine, Rm. 3038, 3501 Hewitt Hall, Irvine, CA 92697-4050. E-mails: guiyuny@uci.edu or zhoug@ uci.edu are readily available in government-run health facilities (with occasional shortages) and in private kiosks. ${ }^{13-16}$ Because of their accessibility, availability, and affordability, over-thecounter (OTC) antimalarial medicines are popular in western Kenya, where malaria transmission remains highest in the country. ${ }^{3,11,13,16}$ Micro-franchising by the private sector is sometimes seen as an approach to make effective healthcare options, such as first-line antimalarials, more accessible to rural populations in need. ${ }^{11,13,17,18}$ However, a previous study found that most adults and children taking OTC medicine did not use multidose antimalarials as recommended. ${ }^{16}$ Non-instructed and out-of-policy use of antimalarials may select for new strains of resistant malaria parasites, ${ }^{7,9,10,19,20}$ which may in turn jeopardize the effectiveness of antimalarial drugs. ${ }^{8,21}$

Although previous studies have investigated malaria treatmentseeking behaviors from both the consumer and provider perspectives, few have studied the temporal changes as malaria epidemiology and case management policy have changed over the last two decades. ${ }^{3,11-13,21-23}$ More importantly, although previous studies investigated the risk factors affecting malaria treatment-seeking behaviors, they rarely examined why some seek treatment at government-run hospitals or health centers (where diagnosis and a pharmacy are available), whereas others self-treat (e.g., buy antimalarials from private shops with or without diagnosis). ${ }^{11,15,16}$ We hypothesize that malaria treatment seeking depends on accessibility, availability, and affordability. Specifically, because ACT drugs are usually available (availability = yes) and free of charge (affordability = yes) in government-run hospitals and health centers, the rule of accessibility means that people who live near hospitals will receive free ACTs more often than people who live far away. For those who live far from hospitals, free antimalarial drugs are not as readily accessible, so they may buy cheap drugs, such as SP, from nearby vendors. It should be noted that SP drugs are usually cheaper than ACTs in private shops, ${ }^{11}$ and $P$. falciparum parasite is highly resistant to SP drugs. ${ }^{24,25}$ The government has no control of the 
sales by private drug vendors either in licensed or unlicensed shops. ${ }^{26,27}$ In addition, previous studies found that distance to health services influences insecticide-treated net (ITN) possession and use. ${ }^{28,29}$

In this study, using data from serial cross-sectional surveys with a random selection of community members and from private retail shops, we aimed to determine the temporal trends and spatial patterns in antimalarial drug availability and usage in western Kenya, so as to better understand the factors affecting malaria treatment-seeking choices. We also conducted spatial analyses to test our hypothesis that people who live near hospitals will receive ACTs more often than people who live far away.

Ethical clearance. Ethical clearance was obtained from the Ethical Review Committee of the Kenya Medical Research Institute, Kenya, and from the Institutional Review Board of the University of California at Irvine, Irvine, CA. For questionnaire surveys, informed oral consent was obtained from heads of households and shopowners. For blood smear sampling, written assent for children ( $<18$ years of age) was obtained by the participants and their parents or guardians.

\section{MATERIALS AND METHODS}

Study area. This study was conducted in western Kenya using questionnaire surveys and cross-sectional parasitological surveys. Community-wide surveys on malaria prevention/ treatment knowledge and antimalarial usage were conducted at four sites: Iguhu in Kakamega County, Emakakha in Vihiga County, Kombewa in Kisumu County, and Marani in Kisii County (Figure 1). Community-based cross-sectional surveys along with cross-sectional malaria prevalence surveys at schools were conducted in randomly selected school-aged children of 6-13 years in Iguhu, Kombewa, and Marani in 2004, 2010, and 2016, respectively. The three study sites were initially selected in 2001 to represent very high (Kombewa), moderate (Iguhu), and low (Marani) transmission intensities. ${ }^{22}$ Surveys on antimalarial drug availability were conducted in 2016 at 10 randomly selected government-run subcounty hospitals or health centers in all counties across the Lake Victoria shore area and adjacent highlands, and 50 private shops within the catchment areas of the 10 hospitals and health centers (Figure 1).

Community-wide surveys of antimalarial usage and malaria knowledge. Cross-sectional community questionnaire surveys of antimalarial usage were conducted in January-March in 2004 and July-August in 2010 and 2016 at three study sites, that is, Iguhu, Kombewa, and Marani. A total of 1,755 households and 9,220 individuals were included over the survey period (Supplemental Appendix Figure S1). For each survey, 200-500 households were randomly selected, covering an area of at least $10 \mathrm{~km}^{2}$ at each site. ${ }^{22}$ For each participating household, the head of the household was informed, and oral consent was obtained. The self-reported malaria cases in the past 3 months (for 2004 and 2010 surveys) or 4 months (for 2016 survey) were obtained from each household, including all family members. The self-reported malaria cases include both confirmed and potentially unconfirmed clinical cases. The names of the antimalarials used were recorded (and confirmed on the spot if available) based on individual usage.

Knowledge of the causes and prevention of malaria surveys were carried out in Iguhu, Kombewa, and Marani areas. In 2004 and 2010, we included questions to assess knowledge of the causes and prevention of malaria; these questions were

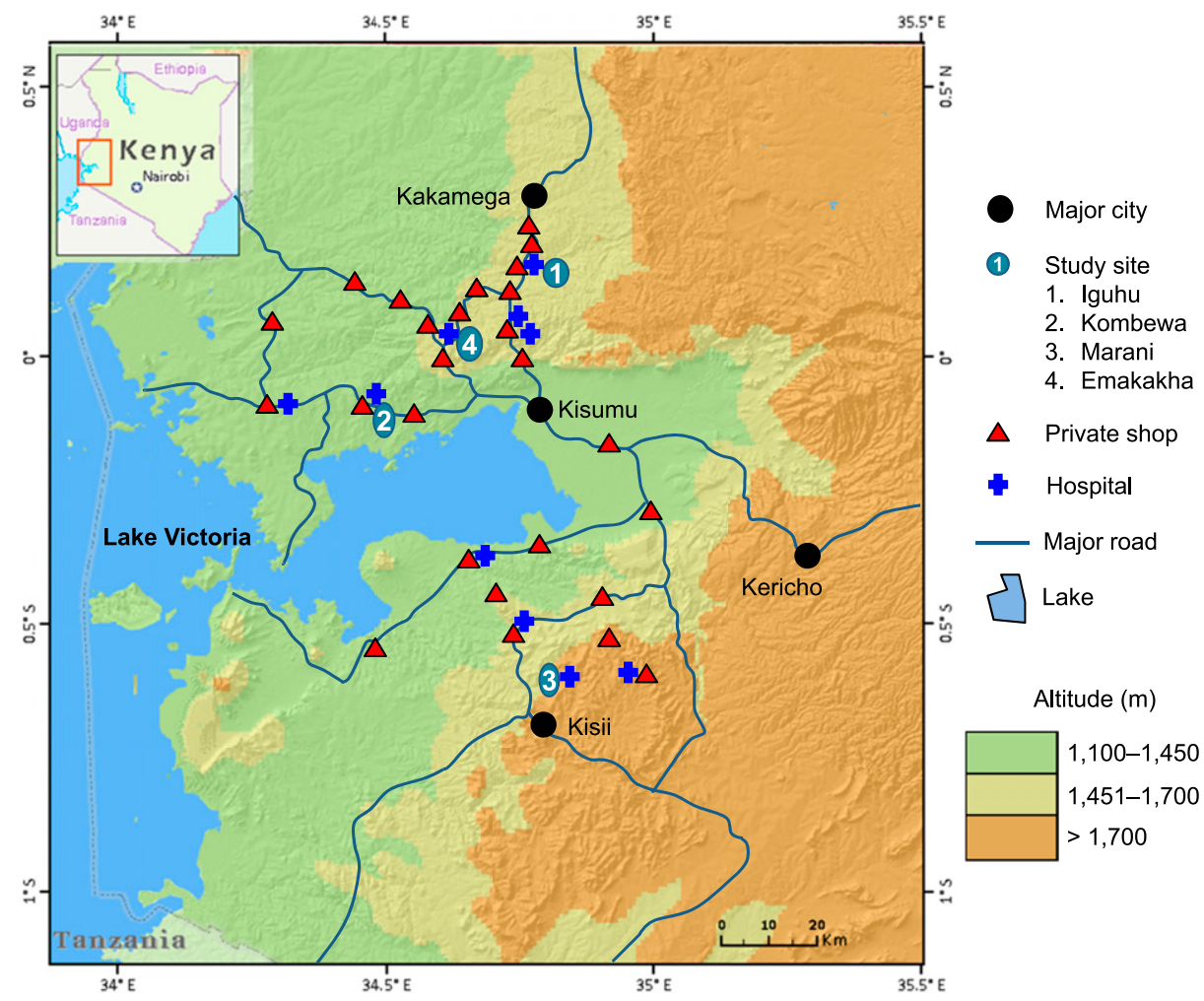

FIGURE 1. Localities of study communities, antimalarial drug survey hospitals, and private shops. This figure appears in color at www.ajtmh.org. 
omitted from the 2016 survey because in 2010 , the vast majority (> 90\%) of surveyed households knew the correct answers.

Treatment-seeking behavior. Household malaria treatmentseeking surveys were conducted in Iguhu, Kombewa, and Marani in 2004 and 2010, and in Iguhu and Emakakha in 2016 (Supplemental Appendix Figure S1). Household malaria treatment seeking was categorized into three classes, that is, seek treatment at government-run hospitals and/or health centers, do a home treatment (i.e., buy drugs from private shops) without seeking treatment at government-run hospitals and/or health centers, or do both. In the 2010 and 2016 surveys, in addition to treatment-seeking locations, the source of antimalarials was also recorded, that is, from pharmacies in the government-run hospitals or health centers or dispensaries, or from private kiosks. To determine the distribution patterns of antimalarial drug usage, we also recorded the global positioning system location of surveyed households. No individual identifiers were asked or recorded during all surveys.

To determine the factors driving treatment-seeking behavior, we collected the following information from each household: parental status; marital status of parents; education level and occupation of the head of household, parents, and/or guardians of the children; household population and agegroup population; house type (wall, roof, floor, eaves, and window screens); insecticide-treated net (ITN; including longlasting insecticidal nets) ownership and use; and wealth indicators, such as ownership of a radio, television, bicycle, motorcycle, car, and sofa set. Education level may affect the household's knowledge about malaria and treatment. ITNs were widespread by 2016, but in 2004 and 2010, coverage was still low, so this information was important.

Malaria parasite prevalence. Cross-sectional parasitological surveys were conducted in randomly selected schoolaged children of 6-13 years in Iguhu, Kombewa, and Marani in 2004, 2010, and 2016, respectively. Blood smear sampling has been described previously. ${ }^{22}$ Briefly, after obtaining assent from participants and parents or guardians, blood samples were collected by the standard finger-prick method, and thin and thick blood smears were prepared for laboratory examination. Parasite species were identified microscopically, and quality control was made by a third technician. ${ }^{22}$

Survey of antimalarial availability. An antimalarial availability survey was conducted at 50 private shops and 10 government-run hospitals and health centers in 2016 across western Kenya, which covers all counties along the Lake Victoria shoreline and adjacent highlands (Figure 1). Privatesector antimalarial drug availability surveys were conducted in the same time frame within the catchment areas of the 10 hospitals (Figure 1). This survey was conducted through convenience sampling. Private kiosks and pharmacies were selected mainly from towns or local marketplaces, where most private shops were located, rather than by randomized selection or systematic census because finding all the private shops was not possible (Figure 1). We selected at most three shops in each town center or marketplace to avoid overweighting the drug types in major town centers. At each shop, after obtaining oral consent from the shopowner, we obtained a list of the names and retail prices of all antimalarials in stock.

Data statistical analysis. Based on efficacy and government policy, for 2004 data, antimalarial drugs were classified as $\mathrm{QN}$, sulfadoxine-pyrimethamine or amodiaquine (AQ) or
CQ, and others (included non-antimalarials or none). For 2010 and 2016 data, drugs were classified as ACT (any ACT drugs including $A L$ unless otherwise specified) or $Q N$ (included combination of ACT or QN with SP or AQ or CQ), SP or AQ or $C Q$, and others (included non-antimalarials and none). Household ITN ownership was defined as the percentage of households that have at least one ITN during the survey over the total households surveyed. Parasite prevalence rate in children was defined as the percentage of children who have plasmodium-positive slide reading by microscopy over the total samples of slides. Clinical case rate was defined as reported malaria episodes per 1,000 people during the survey. We compared changes over time in self-reported malaria (percent of household that reported having malaria) and case rate (episodes per 100 people) using $x^{2}$-tests.

Logistic regression was used to determine the factors affecting treatment-seeking choices. Two models were, respectively, established to determine factors affecting "seek treatment at hospital or health centers" and "home treatment or buy antimalarials from private shop." The independent variables included education level and occupation, household size and number of family members in different age-groups (i.e., 0-4 years, 5-14 years, and 15+ years), wealth index, distance to the nearest major road, and distance to the nearest government-run health center or hospital (excluding dispensaries). Wealth index was established using principal component analysis based on house type, household ownership of sofa set, and number of cows, radios, televisions, bicycles, motorcycles, cars, and cell phones. The case score of the first component was used as the wealth index. The stepwise method was used for variable selection after adjustment by study sites. Data analysis was conducted using R 3.3 (The R Foundation for Statistical Computing, Vienna, Austria).

\section{RESULTS}

Decline in self-reported malaria and improvement in knowledge. Household ITN ownership increased significantly from an average of $19.2 \%$ in 2004 to $68.1 \%$ and $91.4 \%$ in 2010 and 2016, respectively (Table 1). At the household level, selfreported malaria decreased from 100\% (households) in 2004

TABLE 1

Changes in household ITN ownership, percentage of household with self-reported malaria, self-reported clinical malaria case rate, and parasite prevalence rate in children aged 6-13 years

\begin{tabular}{clccc}
\hline \multicolumn{1}{c}{ Site } & \multicolumn{1}{c}{ Indicator } & $2004(\%)$ & $2010(\%)$ & $2016(\%)$ \\
\hline Iguhu & Household ITN ownership & 11.8 & 59.8 & 88.3 \\
& Household malaria rate & 100 & 84.2 & 61.8 \\
& Clinical case rate & 34.0 & 44.2 & 21.1 \\
& Parasite prevalence & 35.3 & 17.4 & 13.5 \\
Kombewa & Household ITN ownership & 38.9 & 78.1 & 91.1 \\
& Household had malaria & 100 & 94.4 & 69.6 \\
& Clinical case rate & 33.2 & 20.7 & 29.6 \\
\multirow{4}{*}{ Marani } & Parasite prevalence & 53.9 & 38.6 & 38.4 \\
& Household ITN ownership & 7.2 & 66.5 & 94.8 \\
& Household had malaria & 100 & 83.7 & 72.5 \\
& Clinical case rate & 35.0 & 29.8 & 28.5 \\
& Parasite prevalence & 8.1 & 0.7 & 4.3 \\
\hline
\end{tabular}

ITN = insecticide-treated net.

${ }^{*}$ Household ITN ownership was defined as the percentage of household who had at least one ITN during the survey over the total households surveyed. Parasite prevalence rate in children was defined as the percentage of children who had Plasmodium-positive slide reading by microscope over the total samples of slides. Clinical case rate was defined as reported malaria episodes per 1,000 people during the survey. 
to $87 \%$ in 2010 and $68 \%$ in 2016 (comparison between 2004 versus 2010 and 2010 versus 2016, $x^{2}$-test, all $P<0.0001$; Table 1). The self-reported malaria case rate declined from $34 \%(1,096 / 3,219)$ in 2004 to $31 \%(848 / 2,762)$ in 2010 and $26 \%(853 / 3,239)$ in 2016 (comparison between 2004 versus 2010 and 2010 versus 2016, $x^{2}$-test, all $P<0.01$; Table 1). Malaria parasite prevalence in children dropped from an average of $32.5 \%$ in 2004 to $18.9 \%$ in 2010 and remained similar by $2016(18.7 \%$; Table 1$)$.

Knowledge about the causes and prevention of malaria improved greatly from 2004 to 2010 (Table 2). Because variations among the three study sites were minimal, the survey data were pooled in subsequent analyses. In 2004 , more than $90 \%$ of the surveyed households were unaware of the causes of malaria or how to prevent malaria (Table 2), whereas in 2010, $93 \%$ of the surveyed households provided the correct answers to what causes malaria and how to prevent malaria (Table 2). It is worth mentioning that during the 2010 surveys, households who were ignorant of the cause or prevention of malaria were all reported in Marani, where malaria prevalence was about $0.7 \%$.

Antimalarial drug availability and usage. Among the 50 private shops, 30 had the government-recommended ACTs (AL) and 48 had other artemisinin-related antimalarials (mostly DHA$P Q$ ), and all 50 shops had either ACT drugs or QN (Supplemental Appendix Table 1). Sulfadoxine-pyrimethamine drugs were also available in 46 of the 50 private shops. There were 23 different brands of artemisinin-related antimalarials and eight different brands of SP drugs (Supplemental Appendix Table S1). Although all of the pharmacies in the surveyed hospitals/health centers had ACTs $(A L)$ in stock, none of them had SP drugs. In private shops, prices for the $\mathrm{WHO}$-recommended $\mathrm{AL}$ were the lowest among ACT drugs (US\$0.8-1.2 per treatment pack), and DHA-PPQ was the most expensive ACT drug (US\$5.0-6.0). Sulfadoxinepyrimethamine drugs (US\$0.2-0.4 per treatment) were less than half the price of $A L$.

Among 2,797 individuals who used antimalarials from the three surveys, $88 \%$ of all antimalarials used in 2004 were SP and $A Q, 8 \%$ were $C Q$, and $4 \%$ were $Q N$ (Figure $2 A$ ). No ACT was used in any of the three sites in 2004. In 2010, the SP/AQ

TABLE 2

Knowledge about the causes and prevention of malaria

\begin{tabular}{|c|c|c|c|c|}
\hline \multirow[b]{2}{*}{ Year of survey } & \multicolumn{2}{|c|}{2004} & \multicolumn{2}{|c|}{2010} \\
\hline & \multicolumn{2}{|c|}{$\begin{array}{c}\text { Number of } \\
\text { households \% }\end{array}$} & \multicolumn{2}{|c|}{$\begin{array}{c}\text { Number of } \\
\text { households \% }\end{array}$} \\
\hline \multicolumn{5}{|l|}{ Causes of malaria } \\
\hline Mosquito bite & 21 & 3.4 & 477 & 93.9 \\
\hline Other causes & $169^{*}$ & 27.4 & 16 & 3.1 \\
\hline Do not know & 427 & 69.2 & 15 & 3.0 \\
\hline Total & 617 & 100 & 508 & 100 \\
\hline \multicolumn{5}{|l|}{ How to prevent } \\
\hline $\begin{array}{l}\text { Mosquito net or indoor } \\
\text { residual spraying }\end{array}$ & 6 & 1.0 & 458 & 90.2 \\
\hline See doctor or buy antimalarial & 26 & 4.2 & 14 & 2.8 \\
\hline Buy local herb medicine & 45 & 7.3 & 0 & 0 \\
\hline Use prophylaxis & 1 & 0.2 & 3 & 0.6 \\
\hline Clean the breeding area & 1 & 0.2 & 0 & 0.0 \\
\hline Other answers & $39+$ & 6.3 & 5 & 1.0 \\
\hline Do not know & 499 & 80.9 & 28 & 5.5 \\
\hline Total & 617 & 100 & 508 & 100 \\
\hline
\end{tabular}

* Premature foods $(n=67)$, cold weather (24), rain (6), witchcraft (9), or a combination of these factors (63).

†Eat matured food $(n=16)$, wear warm clothes (8), a combination of both (4), and unpreventable (11). usage dropped to $39 \%$, ACT usage increased to $48 \%$, and $10 \%$ were QN and 2\% CQ (Figure 2B). In 2016, SP/AQ drugs accounted for only $27 \%$, ACT usage increased to $69 \%$, and $4 \%$ were QN, and no CQ use was reported (Figure 2C). There was also a significant difference in antimalarials obtained from different sources (Figure 3, Supplemental Appendix Table 2). In 2010 , the majority of antimalarials obtained from governmentrun health facilities were ACT drugs (average of $69 \%$ and ranged between $60 \%$ and $80 \%$ ), whereas $~ 80 \%$ of the antimalarials obtained from private shops were $\mathrm{CQ}, \mathrm{SP}$, or $\mathrm{AQ}\left(\mathrm{x}^{2}=355.6\right.$, d.f. $=4, P<0.0001$; Figure 3). Interestingly, no $C Q$ and very few AQ drugs were found in 2016, whereas SP drugs accounted for $28 \%$ of antimalarial drugs obtained from government-run health facilities (note none of the surveyed hospitals reported SP drug in stock) and $24 \%$ of antimalarials from private shops. Artemisinin-based combination therapy was the predominant drug in both places $\left(x^{2}=19.2\right.$, d.f. $=4, P<0.001$; Figure 3$)$.

At the individual level, the majority $(72.9 \%)$ of malaria treatments were ACT or QN in 2016, regardless of the source of antimalarials. However, non-IPTp use of SP drugs was common (20.9\%), and treatment of non-malaria with antimalarials was about $10.1 \%$. Among pregnant women, $94.5 \%$ were provided with either SP (90.9\%) or ACT, whereas only $30.9 \%$ of pregnant women reported having malaria. The rest of them were not provided with IPTp and were not diagnosed with malaria.

Malaria treatment-seeking behavior and driving factors. Malaria treatment-seeking behavior varied from year to year $\left(X^{2}\right.$-test, all $\left.P<0.0001\right)$. In 2004, 27.2\% (168/617) of households sought hospital treatment alone, $40.4 \%$ (249/617) practiced self-treatment without a hospital visit, and $27.7 \%$ (171/617) went to the hospital and practiced self-treatment. In 2010 , the numbers were $44.5 \%$ (226/508) for hospital alone, $2.6 \%(13 / 508)$ for self-treatment alone, and 51.2\% (260/508) for hospital and self-treatment. In 2016, 50.6\% went to the hospital for treatment and $82.0 \%$ of them got medicine from the hospital. About a quarter (24.3\%) of households practiced both home and hospital treatments; $14.5 \%$ used other treatments, mainly pain killers. It is worth noting that the percentage of households who reported to have fever or malaria but did seek any treatment did not change much from $2004(5.7 \%$ or $35 / 617)$ to $2010(6.9 \%$ or $35 / 508)\left(x^{2}=0.71\right.$, d.f. $=1, P=$ $0.399)$; however, this number decreased significantly from 2010 to $2016(0.3 \%$ or $2 / 595)\left(x^{2}=36.30\right.$, d.f. $\left.=1, P<0.0001\right)$. Because of the small number of households who did not seek treatment, non-care-seeking data were not further analyzed.

Further analyses indicated that in 2004, antimalarial usage decreased as the distance to the nearest major road increased (K-S test, $P<0.05$ ). In 2016, the proportion of households using SP drugs increased as the distance to the nearest hospital increased (K-S test, $P<0.05$ ). For SP obtained from hospitals, out-of-policy usage increased with increased distance to the nearest hospital $\left(x^{2}=22.03\right.$, d.f. $\left.=4, P=0.0002\right)$. For SP obtained from private shops, out-of-policy usage increased with increased distance to the nearest major road $\left(x^{2}=1.25\right.$, d.f. $\left.=3, P=0.741\right)$.

Logistic regression revealed that the factors affecting treatment-seeking choices varied over time (adjusted by study sites) (Table 3, Supplemental Appendix Table 3). There were differences in treatment-seeking behavior between study sites during all studies (Table 3). In 2004, households with a better educated household head or with more young children $(<5$ years) were more likely to see doctors. Conversely, households 


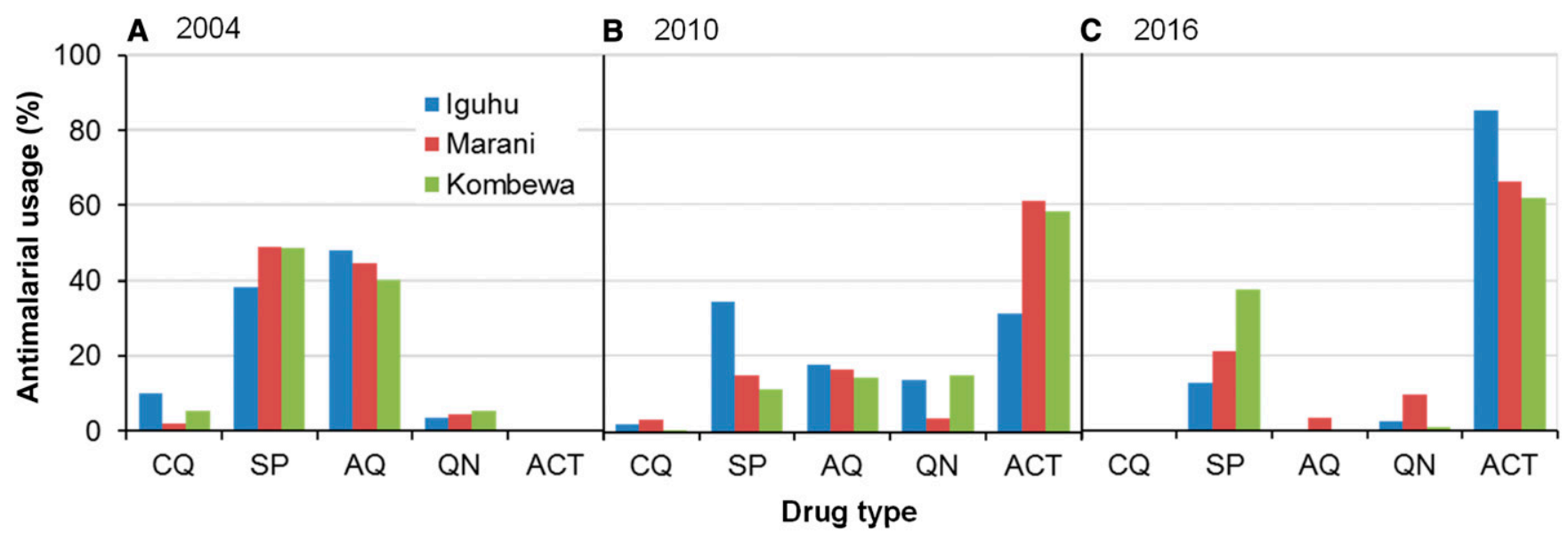

FIGURE 2. Antimalarial drug usage by drug type, study site, and survey year $\left(x^{2}\right.$-test, all $\left.P<0.0001\right)$. ACT = artemisinin-based combination therapy; $\mathrm{AQ}=$ amodiaquine; $\mathrm{CQ}=$ chloroquine; $\mathrm{QN}=$ quinine; $\mathrm{SP}=$ sulphadoxine-pyrimethamine. This figure appears in color at www.ajtmh.org.

located far from hospitals tended to obtain drugs from private shops (Table 3). Likewise, in 2010 and 2016, households that were farther from the main road or hospital tended to practice self-treatment, and households with head as farmers were less likely to go to hospitals (Table 3).

\section{DISCUSSION}

After $10+$ years of intensive antimalaria campaigns that primarily focus on long-lasting insecticidal net use and ACT treatment, our study indicated that ITN ownership has significantly increased, knowledge of malaria prevention has improved, and self-reported malaria has declined in western Kenya. Furthermore, we found that current first-line malaria treatment drugs were available in all government-run hospitals and health centers. Although OTC sales of antimalarial drugs are common in private shops, these private vendors can serve to complement the needs of locals when drugs are out of stock in government-run facilities or access is difficult. ${ }^{30,31}$ However, there is concern about the observations of high rates of out-ofpolicy or non-instructed drug use, especially with SP drugs from private shops, which could lead to the development of new resistant parasites and thwart efforts to fight malaria in the era of malaria elimination. ${ }^{16,19,21}$ A recent study in the same study sites found that genetic markers associated with $P$. falciparum resistance to SP drugs increased to near fixation from 2008 to 2015 , indicating alarming signs of increased SP resistance in western Kenya, ${ }^{32}$ potentially due to increased usage of SP drugs for IPTp-SP or out-of-policy use of SPs. Interestingly, no government health facility had SP in stock during our survey in 2016, which is very surprising given that IPTp policy has been implemented in Kenya for many years. ${ }^{3}$ Intermittent preventive treatment in pregnancy-SP drugs should be provided for free by government-run health facilities, so the reason that no government health facility had SP in stock needs further investigation. With that said, the vast majority (95\%) of surveyed pregnant women were somehow provided with IPTp-SP in 2016. Nonetheless, the impact of out-of-policy drug uses on

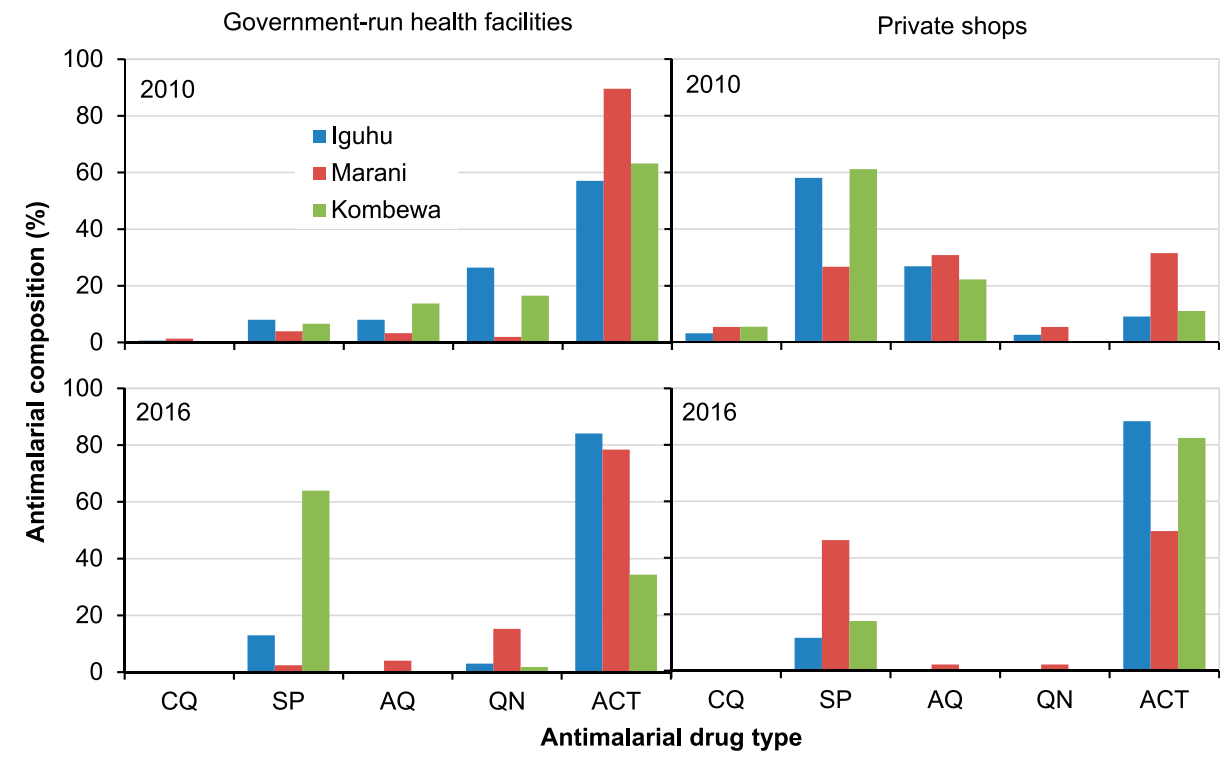

FIGURE 3. Antimalarial drug usage by drug type, source, and study site. $A C T=$ artemisinin-based combination therapy; $A Q=a m o d i a q u i n e ; C Q=$ chloroquine; $\mathrm{QN}$ = quinine; $\mathrm{SP}$ = sulphadoxine-pyrimethamine. This figure appears in color at www.ajtmh.org. 


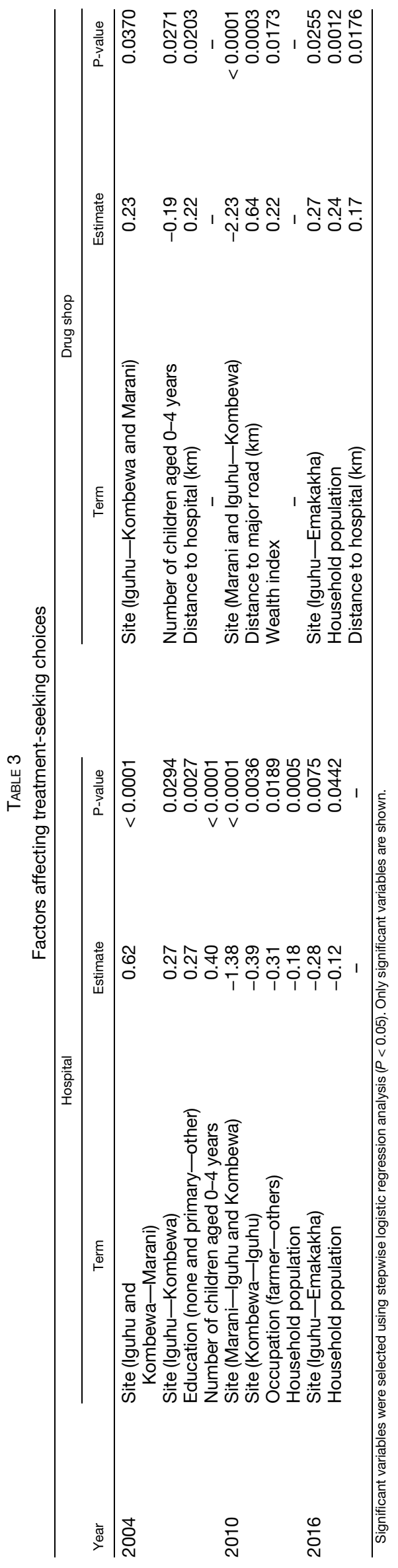

malaria control and on the development of drug resistance must be carefully monitored and evaluated.

Our study found that most (>90\%) of the households have knowledge about the cause and prevention of malaria; however, malaria treatment behavior differed among communities. There was a substantial difference in SP and ACT usage among communities in 2010 and 2016, likely because of both healthcare-seeking behavior and availability of healthcare services in the community. The most striking finding of this study is the gaps between malaria knowledge and treatmentseeking behavior. Although most people in the community have knowledge about malaria treatment, most malaria treatments were ACT or QN, and close to $95 \%$ of pregnant women were provided with IPTp-SP in 2016, and a good proportion $(21 \%)$ of nonpregnant and non-children malaria were treated with SP drug, regardless of the source of antimalarials. This out-of-policy treatment may be partially caused by clinical treatment costs and the availability of healthcare facilities. By the Kenyan government policy, SP should only be used for IPTp-SP. ${ }^{3}$ However, SP (US\$0.2-0.4 per treatment) is 2-4 times cheaper than ACT (US\$0.8-1.2 per treatment) on the market. Although, by policy, ACTs are free of charge in government-run hospitals, it must be a confirmed clinical malaria case. However, laboratory confirmation costs about US\$1.2 in nearly all government-run health facilities. Thus, one has to pay US $\$ 1.2$ to receive the free ACT drugs, and consequently, obtaining antimalarial drugs directly from private shops saves money. Moreover, because SP drugs are available everywhere in private shops and much cheaper than ACT drugs, SP may be an appealing alternative. Last, for those who live far from the government-run hospitals or healthcare centers, buying drugs is also a good option if the sickness is not severe because transportation also costs money.

Among all study sites, Emakakha has the highest coverage of ACT in nearly all treatments in 2016. This phenomenon is likely because of the efforts of community health workers (CHWs), members of the community who work as volunteers to fill the gaps left by shortages of healthcare workers. Community health workers are the entry points for disease prevention and control in the government's new Kenya Community Health Strategy. ${ }^{33}$ They are especially important in areas that are beyond the reach of national healthcare systems. ${ }^{34,35}$ Unfortunately, we have to emphasize that we did not collect any data on the coverage area and how these $\mathrm{CHWs}$ worked in our study area. Emakakha village is about $6 \mathrm{~km}$ from the Emuhaya Health Center (the nearest health center/hospital). The only health service facility in Emakakha village is a dispensary with minimal healthcare service. The involvement of $\mathrm{CHWs}$ who provided additional malaria diagnosis with rapid diagnosis tests (RDTs) and treatment in Ebusiratsi and Emakakha villages may explain the surprisingly high coverage of ACT in the Emakakha area $(\mathrm{H}$. E. Atieli, unpublished data). In this respect, $\mathrm{CHW}$ practice should be encouraged and expanded to areas where health facilities and services are lacking, which is especially important because the expansion of RDTs to CHWs is better using the limited resources of antimalarials in these resource-deficient areas.

Many factors contribute to disease treatment-seeking behavior. ${ }^{12,36}$ Previous studies have found that distance to hospitals/health centers was not associated with delayed treatment. $^{12,14,37}$ However, those studies included all healthcare 
facilities, such as hospitals, health centers, dispensaries, and private clinics, but did not compare self-treatment with hospital treatment. In this study, we separated government-run health centers and hospitals from the other facilities because those are the only places where both diagnosis and free antimalarials are provided. Our results showed that the choice of malaria treatment was clearly determined by availability, accessibility, and affordability. Distance to government-run health centers and hospitals, or to a main road where public transportation is available, is an important determinant. Previous studies found that distance to health services is an important factor affecting distribution of ITNs. ${ }^{28,29}$ This study found that those who lived near hospitals that offer free treatment preferred to seek treatment in hospitals. However, for individuals who live far from hospitals, they preferred to obtain antimalarials from local private shops and most often more affordable drugs such as SP. Unless local health service (e.g., CHW service) is improved, effective malaria case management will remain a problem in rural western Kenya, and this will hamper the malaria control and local elimination efforts.

One of the limitations of this study is the inability to confirm self-reported clinical malaria. It would be ideal to know the diagnostic test results for self-reported clinical malaria; that is, if one has a negative test, then the use of other treatments such as pain killers may not be a problem. However, in 2004, hospital records at the three study areas showed that nearly all malaria cases were unconfirmed but based on clinical symptoms because of the lack of a diagnostic facility; in 2016, about two-thirds of the malaria cases reported from Iguhu and Marani hospitals and all cases reported from Kombewa hospital were confirmed cases (G. Zhou, unpublished data). On the other hand, the use of microscopy or RDTs as standard malaria diagnostic method may further complicate the issue of case diagnosis accuracy and treatment policy. ${ }^{3,38-40}$ Standard diagnostic procedures need further investigation. ${ }^{41-43}$

Another limitation of this study is the convenience sampling of antimalarial drug availability in the private sector. In this study, private kiosks and pharmacies were selected mainly from towns or local marketplaces. Previous studies also found that most private drug shops were located along the road in towns or local marketplaces in Kenya. ${ }^{11,13-16}$ Therefore, the impact of sampling procedure on drug availability may be limited.

Last, age and gender of the person with fever are relevant factors that could be associated with care-seeking behavior. During our field surveys, some of the surveyed households said that they went to the hospital when children were sick. However, they might just buy drugs from private shops when an adult was sick dependent on the severity of the symptoms (G. Zhou, unpublished data). Whether these care-seeking behaviors affect the overall care seeking in the community should be explicitly explored.

Malaria control in western Kenya has achieved great success, but gaps in case management remain. Out-of-policy treatment of malaria is still a concern. Adequate health service facilities are desperately needed to achieve sufficient coverage of effective treatment. Improvement in case management would accelerate progress in malaria elimination in western Kenya in specific and in Africa in general.

Received December 6, 2019. Accepted for publication February 5, 2020.

Published online March 16, 2020.
Note: Supplemental table and figures appear at www.ajtmh.org.

Acknowledgments: We wish to thank all who participated in the field surveys for their assistance with fieldwork. We thank the communities and private drug shopowners and hospitals/health centers for their willingness to participate in this study. The funder has no role in study design; collection, management, analysis, and interpretation of data; writing of the report; or the decision to submit the report for publication. This article is published with permission from the director of Kenya Medical Research Institute.

Financial support: This study was funded by the National Institutes of Health (R01 A1050243, D43 TW01505, R01 Al094580, and U19 Al129326).

Authors' addresses: Guofa Zhou, Elizabeth Hemming-Schroeder, Ming-Chieh Lee, and Guiyun Yan, Program in Public Health, University of California at Irvine, Irvine, CA, E-mails: zhoug@uci.edu, ehemming@ uci.edu, mingchil@uci.edu, guiyuny@uci.edu, and guiyuny@uci.edu. Maxwell Gesuge and Andrew K. Githeko, Kenya Medical Research Institute, Kisumu, Kenya, E-mails: machani.maxwe2011@gmail.com and githeko@yahoo.com. Yaw A. Afrane, College of Health Sciences, University of Ghana, Accra, Ghana, E-mail: yaw_afrane@yahoo.com. Harrysone E. Atieli, Department of Public Health, Maseno University, Maseno, Kenya, E-mail: etemesi2012@yahoo.com.

\section{REFERENCES}

1. World Health Organization, 2019. World Malaria Report 2019. Geneva, Switzerland: WHO.

2. Chuma J et al., 2009. Reviewing the literature on access to prompt and effective malaria treatment in Kenya: implications for meeting the Abuja targets. Malar J 8: 243.

3. Ministry of Public Health and Sanitation, 2010. National Guideline for the Diagnosis, Treatment and Prevention of Malaria in Kenya. Nairobi, Kenya: Republic of Kenya.

4. Centers for Disease Control and Prevention, 1978. Chloroquineresistant malaria acquired in Kenya and Tanzania, Denmark, Georgia, New York. MMWR 27: 463-464.

5. Esposito R, Orlando G, Crocchiolo P, 1983. In vitro confirmation of clinical resistance of Plasmodium falciparum to chloroquine in Kenya. Trans R Soc Trop Med Hyg 77: 421.

6. Brandling-Bennett AD, Oloo AJ, Khan B, Watkins WM, 1988. Failure of erythromycin to improve chloroquine treatment of Plasmodium falciparum malaria in Kenya. Trans $R$ Soc Trop Med Hyg 82: 363-365.

7. Sibley CH, Hyde JE, Sims PF, Plowe CV, Kublin JG, Mberu EK, Cowman AF, Winstanley PA, Watkins WM, Nzila AM, 2001. Pyrimethamine-sulfadoxine resistance in Plasmodium falciparum: what next? Trends Parasitol 17: 582-588.

8. Anabwani GM, Esamai FO, Menya DA, 1996. A randomised controlled trial to assess the relative efficacy of chloroquine, amodiaquine, halofantrine and fansidar in the treatment of uncomplicated malaria in children. East Afr Med J 73: 155-158.

9. Ogutu BR, Smoak BL, Nduati RW, Mbori-Ngacha DA, Mwathe F, Shanks GD, 2000. The efficacy of pyrimethamine-sulfadoxine (Fansidar) in the treatment of uncomplicated Plasmodium falciparum malaria in Kenyan children. Trans $R$ Soc Trop Med Hyg 94: 83-94.

10. Obonyo CO, Ochieng F, Taylor WR, Ochola SA, Mugitu K, Olliaro $P$, ter Kuile F, Oloo AJ, 2003. Artesunate plus sulfadoxinepyrimethamine for uncomplicated malaria in Kenyan children: a randomized, double-blind, placebo-controlled trial. Trans $R$ Soc Trop Med Hyg 97: 585-591.

11. Dixit A, Lee M-C, Goettsch B, Afrane Y, Githeko AK, Yan G, 2016. Discovering the cost of care: consumer, provider, and retailer surveys shed light on the determinants of malaria healthseeking behaviours. Malar J 15: 179.

12. The malERA Consultative Group on Drugs Review, 2011. A research agenda for malaria eradication: drugs. PLoS Med 8: e1000402.

13. Smith N, Obala A, Simiyu C, Menya D, Khwa-Otsyula B, O'Meara WP, 2011. Accessibility, availability and affordability of antimalarials in a rural district in Kenya after implementation of a national subsidy scheme. Malar J 10: 316. 
14. Watsierah CA, Ouma C, 2014. Access to artemisinin-based combination therapy (ACT) and quinine in malaria holoendemic regions of western Kenya. Malar J 13: 290.

15. Rusk A, Highfield L, Wilkerson JM, Harrell M, Obala A, Amick B, 2016. Geographically-weighted regression of knowledge and behaviour determinants to anti-malarial recommending and dispensing practice among medicine retailers in western Kenya: capacitating targeted interventions. Malar J 15: 562.

16. Abuya TO, Mutemi W, Karisa B, Ochola SA, Fegan G, Marsh V, 2007. Use of over-the-counter malaria medicines in children and adults in three districts in Kenya: implications for private medicine retailer interventions. Malar J 6: 57.

17. Chiguzo AN, Mugo RW, Wacira DG, Mwenda JM, Njuguna EW, 2008. Delivering new malaria drugs through grassroots private sector. East Afr Med J 85: 425-431.

18. Galactionova K, Tediosi F, de Savigny D, Smith T, Tanner M, 2015. Effective coverage and systems effectiveness for malaria case management in sub-Saharan African countries. PLoS One 10: e0127818.

19. Juma DW et al., 2012. Trends in drug resistance codons in Plasmodium falciparum dihydrofolate reductase and dihydropteroate synthase genes in Kenyan parasites from 2008 to 2012. Malar J 13: 250.

20. Maiga $\mathrm{H}$ et al., 2016. Seasonal malaria chemoprevention with sulphadoxine-pyrimethamine and amodiaquine selects Pfdhfrdhps quintuple mutant genotype in Mali. PLoS One 11: e0162718.

21. Siringi $S, 2001$. Over-the-counter sale of antimalaria drugs stalls Kenyan disease strategy. Lancet 357: 1862.

22. Zhou G, Afrane YA, Vardo-Zalik AM, Atieli H, Zhong D, Wamae P, Himeidan YE, Minakawa N, Githeko AK, Yan G, 2011. Changing patterns of malaria epidemiology between 2002 and 2010 in western Kenya: the fall and rise of malaria. PLoS One 6: e20318.

23. Trape J-F et al., 2014. The rise and fall of malaria in a west African rural community, Dielmo, Senegal, from 1990 to 2012: a 22 year longitudinal study. Lancet Infect Dis 14: 476-488.

24. Lucchi NW, Okoth SA, Komino F, Onyona P, Goldman IF, Ljolje D, Shi YP, Barnwell JW, Udhayakumar V, Kariuki S, 2015. Increasing prevalence of a novel triple-mutant dihydropteroate synthase genotype in Plasmodium falciparum in western Kenya. Antimicrob Agents Chemother 59: 3995-4002.

25. Mbonye AK, Birungi J, Yanow SK, Shokoples S, Malamba S, Alifrangis M, Magnussen P, 2015. Prevalence of Plasmodium falciparum resistance markers to sulfadoxine-pyrimethamine among pregnant women receiving intermittent preventive treatment for malaria in Uganda. Antimicrob Agents Chemother 59: 5475-5482.

26. Bell DJ, Nyirongo SK, Mukaka M, Zijlstra EE, Plowe CV, Molyneux ME, Ward SA, Winstanley PA, 2008. Sulfadoxine-pyrimethamine-based combinations for malaria: a randomised blinded trial to compare efficacy, safety and selection of resistance in Malawi. PLoS One 3: e1578.

27. Larson PS, Mathanga DP, Campbell CH Jr., Wilson ML, 2012. Distance to health services influences insecticide-treated net possession and use among six to 59 month-old children in Malawi. Malar J 11: 18.

28. Prudhomme O'Meara W, Smith N, Ekal E, Cole D, Ndege S, 2011. Spatial distribution of bednet coverage under routine distribution through the public health sector in a rural district in Kenya. PLoS One 6: e25949.
29. Zongo I, Dorsey G, Rouamba N, Dokomajilar C, Séré Y, Rosenthal PJ, Ouédraogo JB, 2007. Randomized comparison of amodiaquine plus sulfadoxine-pyrimethamine, artemether-lumefantrine, and dihydroartemisinin-piperaquine for the treatment of uncomplicated Plasmodium falciparum malaria in Burkina Faso. Clin Infect Dis 45: 1453-1461.

30. Minstry of Public Health and Sanitation of Kenya, 2009. Kenya Malaria Programme Performance Review 2009. Nairobi, Kenya: Ministry of Public Health and Sanitation.

31. McCombie SC, 1996. Treatment seeking for malaria: a review of recent research. Soc Sci Med 43: 933-945.

32. Hemming-Schroeder $E$ et al., 2018. Impacts of antimalarial drugs on Plasmodium falciparum drug resistance markers, western Kenya, 2003-2015. Am J Trop Med Hyg 98: 692-699.

33. Ministry of Health of Kenya, 2006. Taking the Kenya Essential Package for Health to the Community: A Strategy for the Delivery of Level One Services. Nairobi, Kenya: Ministry of Health.

34. Oliver M, Geniets A, Winters N, Rega I, Mbae SM, 2015. What do community health workers have to say about their work, and how can this inform improved programme design? A case study with CHWs within Kenya. Glob Health Action 8: 27168.

35. Adam MB, Dillmann M, Chen M-K, Mbugua S, Ndung'u J, Mumbi P, Waweru E, Meissner P, 2014. Improving maternal and newborn health: effectiveness of a community health worker program in rural Kenya. PLoS One 9: e104027.

36. Ingabire CM et al., 2016. Determinants of prompt and adequate care among presumed malaria cases in a community in eastern Rwanda: a cross sectional study. Malar J 15: 227.

37. Feikin DR, Nguyen LM, Adazu K, Ombok M, Audi A, Slutsker L, Lindblade KA, 2009. The impact of distance of residence from a peripheral health facility on pediatric health utilisation in rural western Kenya. Trop Med Int Health 14: 54-61.

38. World Health Organization, 2015. Guidelines for the Treatment of Malaria. Geneva, Switzerland: WHO. Available at: http:// apps.who.int/iris/bitstream/10665/162441/1/9789241549127_ eng.pdf. Accessed Noverber 28, 2019.

39. Mahende C, Ngasala B, Lusingu J, Yong T-S, Lushino P, Lemnge M, Mmbando B, Premji Z, 2016. Performance of rapid diagnostic test, blood-film microscopy and PCR for the diagnosis of malaria infection among febrile children from Korogwe district, Tanzania. Malar J 15: 391.

40. Kang JM et al., 2017. Comparison of the diagnostic performance of microscopic examination with nested polymerase chain reaction for optimum malaria diagnosis in upper Myanmar. Malar J 16: 119.

41. Zhou G, Afrane YA, Malla S, Githeko AK, Yan G, 2015. Active case surveillance, passive case surveillance and asymptomatic malaria parasite screening illustrate different age distribution, spatial clustering and seasonality in western Kenya. Malar J 14: 41.

42. Afrane YA, Zhou G, Githeko AK, Yan G, 2013. Utility of health facility-based malaria data for malaria surveillance. PLoS One 8: e54305.

43. Kapesa A, Kweka EJ, Zhou G, Atieli HE, Kamugisha E, Mazigo HD, Ngallaba SE, Githeko AK, Yan G, 2018. Utility of passive malaria surveillance in hospitals as a surrogate to community infection transmission dynamics in western Kenya. Arch Public Health 76: 39. 